\title{
Linfoma primario de las efusiones con afectación extracavitaria multisistémica en HIV negativo diagnosticado mediante autopsia
}

\author{
Primary effusion lymphoma with multisystemic extracavitary involvement in HIV negative \\ diagnosed by autopsy

\section{Linfoma primário de efusões com envolvimento extracavitário multissistêmico em HIV negativo diagnosticado por autópsia}

\author{
Martín Milanesio ${ }^{1-2}$, Ana Victoria Sánchez ${ }^{1-3}$, Luciana Guanchiale ${ }^{1-4}$, Ruth Lía Kaplan ${ }^{1-5}$.
}

Los Linfomas son proliferaciones malignas de linfocitos. El Linfoma primario de las efusiones es un tipo de Linfoma que se caracteriza por afectar distintas cavidades del organismo, como la cavidad pleural, generando acumulación de líquido en las mismas. Este tipo de Linfoma afecta preferentemente a personas infectadas por el virus de la inmunodeficiencia humana y rara vez se extiende más allá de las membranas serosas. En el siguiente manuscrito describimos el caso de un varón añoso HIV negativo que en la autopsia se diagnosticó Linfoma primario de las efusiones con afectación extracavitaria extensa.

\section{Conceptos clave:}

\section{¿Qué se sabe sobre el tema?}

El Linfoma primario de las efusiones es un Linfoma no Hodgkin que afecta principalmente a personas infectadas por virus de la inmunodeficiencia humana (HIV). Se caracteriza por la presencia de derrame linfomatoso maligno en las diferentes cavidades serosas del cuerpo siendo la afectación extracavitaria extremadamente infrecuente..

\section{¿Qué aporta este trabajo?}

Presentamos el caso de un varón HIV negativo con derrame pleural y ascitis que mediante la autopsia se llegó al diagnóstico de Linfoma primario de las efusiones con afectación extracavitaria multisistémica.

1- Hospital Privado Universitario de Córdoba; Argentina. 2- ORCID: https://orcid.org/0000-0002-9521-3601. E-mail de contacto: mmilanesio5@gmail.com.

3- E-mail de contacto: anavictoriasanchez2@gmail.com

4- E-mail de contacto: lucianaguanchiale@hotmail.com

5- E-mail de contacto: ruth.kaplan@hospitalprivado.com.ar

\section{Resumen:}

Introducción: El Linfoma primario de las efusiones es un Linfoma no Hodgkin de alto grado y escasa frecuencia producido por el virus Herpes Humano 8. Afecta principalmente a individuos infectados por el virus de la inmunodeficiencia humana (HIV), aunque también se ha descripto en HIV negativos. Se caracteriza por la presencia de derrame linfomatoso maligno en las distintas cavidades serosas siendo la cavidad pleural la más afectada. La afectación fuera de las serosas es muy poco frecuente. El diagnóstico se realiza por análisis del líquido acumulado en las serosas o mediante biopsia de las mismas demostrando la presencia de linfocitos neoplásicos característicos. El tratamiento consiste en distintos regímenes de quimioterapia asociados a terapia antirretroviral en pacientes HIV positivos. El pronóstico de esta enfermedad es pobre, con una supervivencia de escasos meses posteriores al diagnóstico. Metodología: Presentamos el caso de un varón de 83 años, HIV negativo, con derrame pleural izquierdo y ascitis, observándose un líquido con predominio de mononucleares sin presencia de células malignas al examen citológico. Resultados: El paciente falleció y mediante la autopsia se llegó al diagnóstico de Linfoma primario de las efusiones, observándose además afectación extracavitaria multisistémica. Conclusión: Si bien el Linfoma primario de las efusiones afecta preferentemente a individuos HIV positivos, la ausencia de infección por HIV no debe descartar la enfermedad. EI compromiso extracavitario debe ser buscado aún en pacientes asintomáticos.

Palabras clave: linfoma de efusión primaria; linfoma no Hodgkin; derrame pleural; ascitis.

\section{Abstract:}

Introduction: Primary effusion lymphoma is a low-frequency and high-grade nonHodgkin lymphoma caused by the Human Herpes virus 8. It mainly affects individuals infected with the human immunodeficiency virus (HIV), although it has also been described in HIV-negative cases. It is characterized by the presence of a malignant lymphomatous effusion in the different serous cavities, the pleural cavity being the most affected. Involvement outside the serosae is very rare. Diagnosis is made by analysis of the fluid accumulated in the serosae or by biopsy of the same, demonstrating the presence of characteristic neoplastic lymphocytes. Treatment consists of different chemotherapy regimens associated with antiretroviral therapy in HIV-positive patients. The prognosis of this disease is poor, with a survival of a few months after diagnosis. Methodology: We present the case of an 83-year-old man, HIV negative, with left pleural effusion and ascites, observing a fluid with a predominance of mononuclear cells without the presence of malignant cells on cytological examination. Results: The patient died and the autopsy made the diagnosis of Primary effusion lymphoma, also observing multisystemic extracavitary involvement. Conclusion: Although Primary effusion lymphoma preferentially affects HIV-positive individuals, the absence of HIV infection should not rule out the disease. Extracavitary involvement should be sought even in asymptomatic patients.

Keywords: lymphoma, primary effusion; lymphoma, non-Hodgkin; pleural effusion; ascites.

\section{Resumo:}

Introdução: O linfoma de derrame primário é um linfoma não Hodgkin de alto grau raro, causado pelo vírus do herpes humano 8. Afeta principalmente indivíduos infectados com o vírus da imunodeficiência humana (HIV), embora também tenha sido descrito em casos HIV negativos. É caracterizada pela presença de derrame linfomatoso maligno nas diferentes cavidades serosas, sendo a cavidade pleural a mais acometida. $O$ envolvimento fora da serosa é muito raro. O diagnóstico é feito pela análise do líquido acumulado na serosa ou por biópsia da mesma, demonstrando a presença de linfócitos neoplásicos característicos. O tratamento consiste em diferentes regimes de quimioterapia associados à terapia antirretroviral em pacientes HIV-positivos. O prognóstico da doença é ruim, com sobrevida de alguns meses após o diagnóstico. Metodologia: Apresentamos o caso de um homem de 83 anos, HIV negativo, com derrame pleural à esquerda e ascite, observando ao exame citológico um líquido com predomínio de células mononucleares sem a presença de células malignas. Resultados: O paciente foi a óbito e a autópsia fez o diagnóstico de linfoma primário de derrames, observando também acometimento extracavitário multissistêmico. Conclusão: Embora o linfoma primário afete preferencialmente indivíduos HIV-positivos, a ausência de infecção pelo HIV não deve excluir a doença. O envolvimento extracavitário deve ser procurado mesmo em pacientes assintomáticos. 


\section{INTRODUCCIÓN}

El Linfoma primario de las efusiones (LEP) es un Linfoma no Hodgkin (LNH) tipo $B$ de alto grado producido por el virus Herpes Humano 8 (VHH- 8) que afecta principalmente a personas infectadas por el virus de la inmunodeficiencia humana (HIV) ${ }^{(1,2)}$.

Presenta dos variantes. La más frecuente denominada clásica, es en la cual se produce derrame linfomatoso maligno en las distintas cavidades corporales con síntomas derivados por el compromiso de las mismas siendo muy poco frecuente el compromiso extracavitario. La otra variante es la extracavitaria, que presenta compromiso fuera de las cavidades serosas sin compromiso de las mismas, siendo este último un criterio de exclusión para su diagnóstico(3-6).

El diagnóstico se realiza mediante el análisis del líquido extraído de la cavidad serosa comprometida. Se caracteriza por ser un exudado a predominio de mononucleares con linfocitos que presentan una morfología e inmunofenotípo distintivo( ${ }^{(7)}$. Un criterio absoluto para el diagnóstico es la presencia de ADN del VHH-8 en el núcleo de los linfocitos neoplásicos ${ }^{(1)}$.

El tratamiento se basa en diferentes regímenes de quimioterapia asociados a terapia antirretroviral (TARV) en caso de individuos HIV positivos.

El pronóstico de la enfermedad es malo, con una mediana de supervivencia global de 6 meses ${ }^{(8)}$.

A continuación presentamos el caso de un varón HIV negativo que presentó un LEP con compromiso extracavitario multisistémico diagnosticado mediante autopsia.

\section{CASO CLÍNICO}

Varón de 83 años, con antecedentes de fibrilación auricular crónica, anticoagulado con apixabán, accidente cerebro vascular isquémico diez años previos al cuadro actual, estenosis uretral, infecciones urinarias a repetición y enfermedad renal crónica.

Consultó por fiebre y disnea de 48 horas de evolución. Hacía dos meses que presentaba anorexia, astenia y distensión abdominal, con empeoramiento en la última semana. Al ingreso se constató temperatura de $38^{\circ} \mathrm{C}$, tensión arterial de $80 / 40 \mathrm{~mm}-\mathrm{Hg}$, frecuencia cardíaca de 80 latidos/minuto, frecuencia respiratoria de 20 respiraciones/minuto y saturación de hemoglobina aire ambiente del $90 \%$ que corregía a $94 \%$ con cánula nasal a 2 litros $/ \mathrm{min}$. Al examen físico estaba somnoliento, sin signos de foco ni meníngeos. Presentaba palidez generalizada, edemas hasta los muslos y distensión abdominal asociada a matidez supraumbilical de concavidad superior. Se auscultaba hipoventilación en la base pulmonar izquierda.

El valor de sus glóbulos blancos era de $7.000 \mathrm{cel} / \mu \mathrm{L}(4.600$ neutrófilos segmentados/ $\mu \mathrm{L}, 2.100$ linfocitos $/ \mu \mathrm{L}, 100$ eosinófilos $/ \mu \mathrm{L}$, 200 monocitos $/ \mu \mathrm{L}$ ), hemoglobina $11.5 \mathrm{~g} / \mathrm{dl}$ normocítica normocrómica y plaquetas $127.000 \mathrm{cel} / \mu \mathrm{L}$. La creatinina era de 2 $\mathrm{mg} / \mathrm{dl}$ (valores habituales de $1.5 \mathrm{mg} / \mathrm{dl}$ ), proteína $\mathrm{C}$ reactiva de 12.2 $\mathrm{mg} / \mathrm{dl}$ (normal hasta $0.6 \mathrm{mg} / \mathrm{dl}$ ), procalcitonina $0.25 \mathrm{ng} / \mathrm{ml}$ (normal hasta $0.5 \mathrm{ng} / \mathrm{ml}$ ), LDH $3005 \mathrm{U} / \mathrm{l}$ (normal hasta $460 \mathrm{U} / \mathrm{l}$ ). lonograma y hepatograma normales.

Se solicitó tomografía de cráneo donde no se apreciaron lesiones agudas, y tomografía tóraco-abdominal en la que se observó derrame pleural izquierdo masivo sin lesiones en parénquima y abundante ascitis asociado a hígado atrófico (Imagen 1).

Se realizó toracocentesis y se obtuvo un líquido tipo exudado según criterios de light, con 10.760 células (80\% de mononucleares). La tinción de Gram y bacilos ácido alcohol resistentes (BAAR) y el cultivo para gérmenes comunes, hongos y micobacterias fueron negativos. En el análisis citológico no se vieron células malignas. También se realizó punción del líquido ascítico obteniéndose un líquido con GASA menor a 1.1 , con 11.279 células $(80 \%$ de mononucleares), LDH 4039 U/l. La tinción de Gram y BAAR y el cultivo para gérmenes comunes, hongos y micobacterias fueron negativos. El análisis citológico no demostró la presencia de células malignas.

Las serologías virales (HIV, hepatitis B, hepatitis C), Mantoux y marcadores oncológicos (CEA, CA 19-9 Y PSA) fueron negativos.

El síndrome ascítico edematoso fue refractario al tratamiento diurético y requirió punciones repetidas, sin realizar análisis del mismo en las punciones posteriores. Tras 10 días de internación presentó hemorragia digestiva baja con descompensación hemodinámica. Los familiares decidieron no realizar maniobras invasivas produciéndose el deceso del paciente.

Se realizó autopsia en la que se observó compromiso por linfocitos neoplásicos de aspecto anaplásico y plasmablástico del parénquima pulmonar, venas pulmonares, cápsula de bazo e hígado, vejiga, uréter, ganglios $y$ tubo digestivo (Imagen $2 \mathrm{~A}$ y $\mathrm{B}$ ). Inmunofenotípicamente eran positivos para CD30 y CD138 (Imagen 2B) y negativos para CD3, CD79a, CD20 (Imagen 2C), kappa, lambda y PAX5. La inmunohistoquímica para $\mathrm{VHH}-8$ fue positiva en el núcleo de los linfocitos neoplásicos (Imagen 2D), llegándose al diagnóstico de LEP. La inmunohistoquímica para Virus Epstein-Barr (VEB) fue negativa.

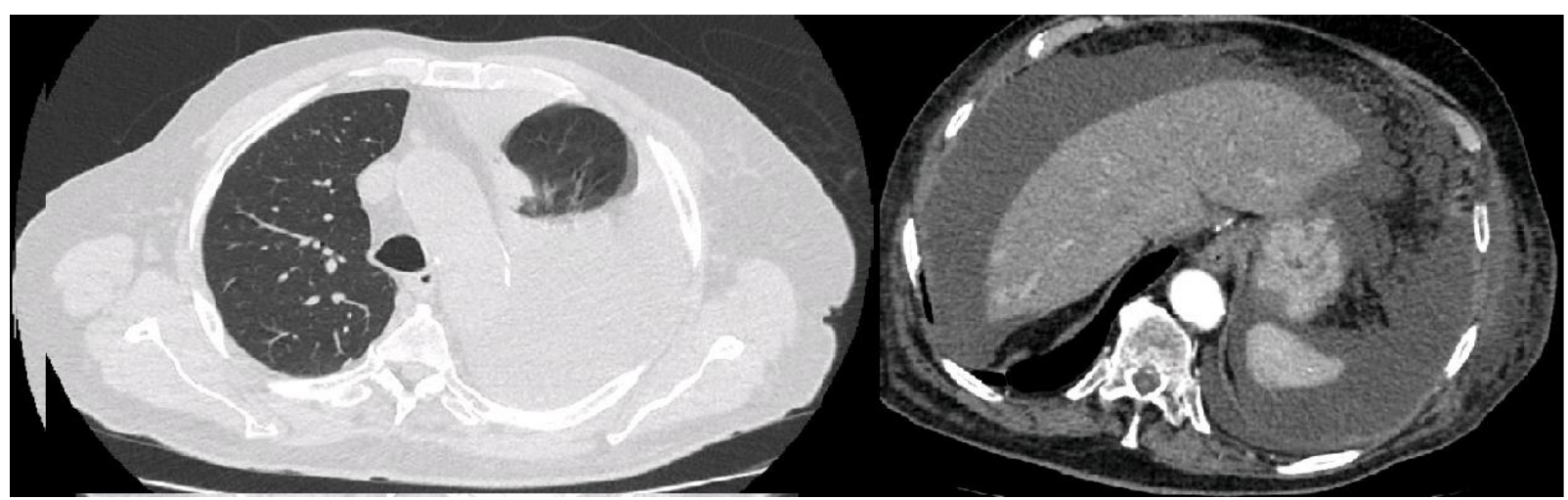

Imagen $\mathrm{N}^{\circ}$. A la izquierda tomografía axial computada (TAC) de tórax en corte axial con ventana pulmonar mostrando derrame pleural izquierdo masivo. A la derecha TAC de abdomen en corte axial con ventana de partes blandas mostrando importante cantidad de ascitis junto con hígado atrófico. 


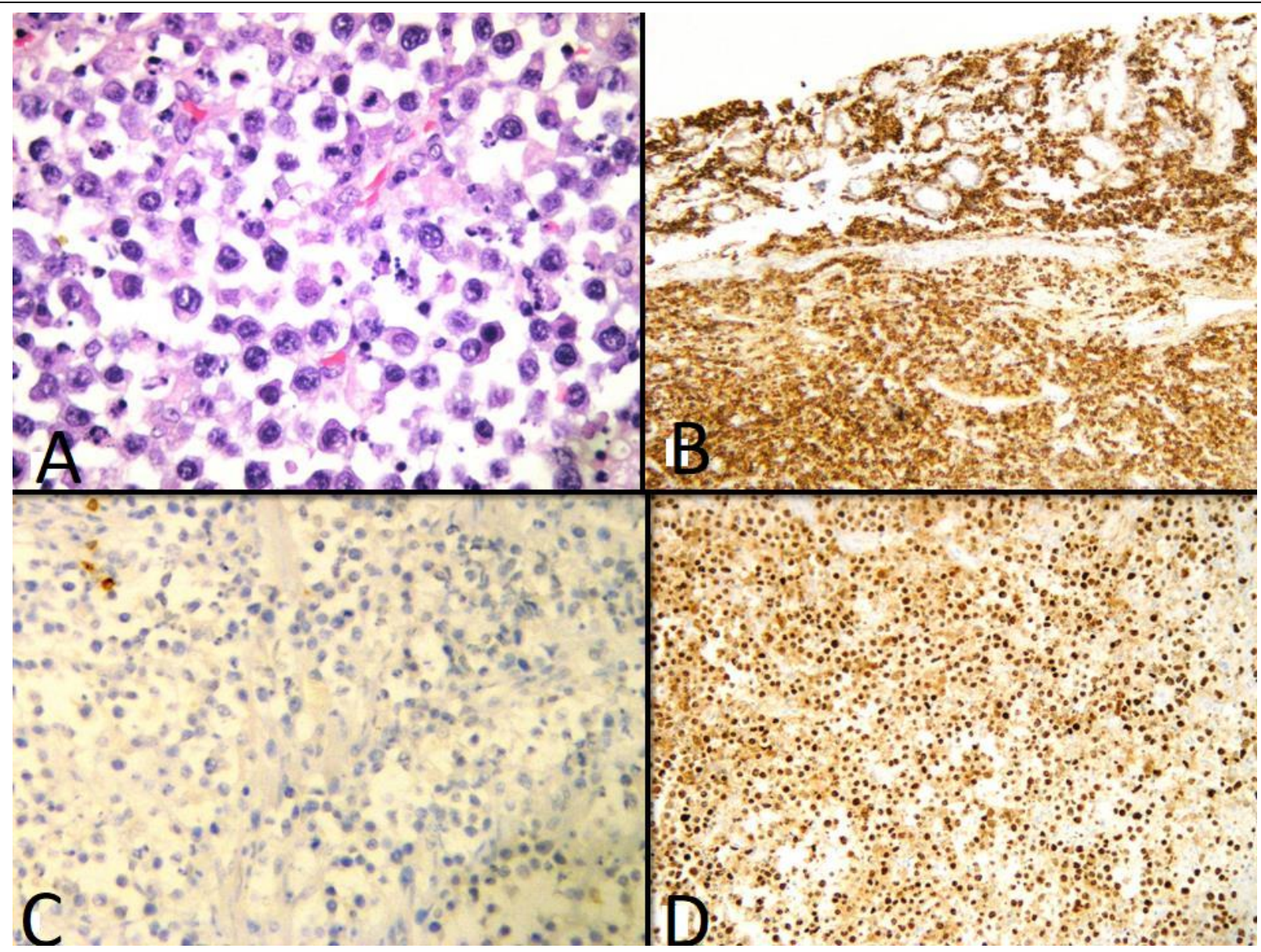

Imagen №2. A. Linfoma primario de las efusiones (LEP). Masa sólida en ganglio linfático. Células grandes con aspecto anaplásico y plasmablástico (Hematoxilina y Eosina 400x). B. Infiltración de pared de tubo digestivo con fuerte expresión del marcador CD138 por inmunohistoquímica demostrando un fenotipo de células B diferenciadas terminal (tinción inmunohistoquímica 400x). C. Ausencia de tinción de marcadores pan-células B, como CD20, característico de este linfoma (tinción inmunohistoquímica 400x). D. Los núcleos de las células neoplásicas son positivos para la proteína latente asociada a VHH-8 LANA1, estableciendo el diagnóstico y diferenciándolo de otros linfomas de células grandes B con diferenciación plasmablástica (tinción inmunohistoquímica 400x).

\section{DISCUSIÓN}

EI LEP representa menos del $1 \%$ de todos los linfomas ${ }^{(9)}$. Es seis veces más frecuentes en varones $^{(9)}$ y afecta principalmente individuos HIV positivo(2), aunque se ha descripto en pacientes infectados por virus de hepatitis $\mathrm{C}^{(10)}$ y $\mathrm{B}^{(11)}$, posterior al trasplante de órganos sólidos ${ }^{(12)}$ y en ancianos sin una afección subyacente ${ }^{(13)}$. En dos series que compararon las diferencias entre LEP en pacientes HIV positivos y HIV negativos se pudo observar que los pacientes HIV negativos presentaban una mayor edad al momento del diagnóstico ${ }^{(1,14)}$._Nuestro paciente no presentaba ninguno de los estados de inmunodepresión citados anteriormente. Es probable que la edad y las comorbilidades incidieran en el desarrollo de la enfermedad, como ocurre en pacientes con LEP HIV negativos ${ }^{(14)}$

La característica distintiva del LEP es la presencia de derrames linfomatosos malignos en las diferentes cavidades serosas, siendo la pleura la más afectada ${ }^{(1,15)}$, seguida por peritoneo y pericardio respectivamente. Aunque pueden afectarse de manera simultánea, lo más frecuente es que sólo se afecte una de ellas ${ }^{(1,15)}$. La sintomatología depende de la cavidad comprometida, siendo la disnea y la distensión abdominal los síntomas más frecuentes debido al compromiso de la pleura y el peritoneo respectivamente. En nuestro caso se presentó de manera concomitante la afectación de la pleura y el peritoneo, manifestando síntomas del compromiso de ambas.

Aunque la afección extracavitaria es algo infrecuente en la variante clásica, las células neoplásicas pueden diseminarse desde una cavidad hacia sitios extracavitarios. Se han descripto casos de afección del tubo digestivo, parénquima pulmonar, ganglios, piel y laringe ${ }^{(15)}$. En nuestro paciente el inicio del cuadro con manifestaciones abdominales y respiratorias nos hace pensar que el foco de la neoplasia se inició en la pleura y el peritoneo con posterior diseminación hacia los demás órganos extracavitarios. Exceptuando la hemorragia digestiva, que consideramos fue por compromiso del tubo digestivo, el paciente no manifestó síntomas de las otras afecciones extracavitarias encontradas en la autopsia. Nos planteamos el interrogante de que el compromiso extracavitario exista y sea más frecuente de lo pensado en pacientes con LEP, pero al no dar síntomas ni verse en las imágenes, pueda pasar desapercibido. En nuestro caso la autopsia permitió evidenciar el extenso compromiso extracavitario que clínicamente no fue sospechado.

EI diagnóstico de LEP se realiza mediante el análisis del líquido extraído de la cavidad comprometida. El mismo es un exudado con abundante celularidad a predominio de mononucleares. Morfológicamente los linfocitos neoplásicos se caracterizan por ser grandes, con núcleos irregulares y nucléolos prominentes ${ }^{(1)}$. Inmunofenotípicamente no presentan marcadores clásicos de células $B(C D 19, C D 20, C D 79 a$,) ni de células $T$ (CD3, CD4 y CD8). Son positivos para marcadores de linfocitos activados (CD30) y de células plasmáticas (CD138) ${ }^{(5)}$. Es condición necesaria para el diagnóstico la presencia de ADN del $\mathrm{VHH}-8$ en el núcleo de los linfocitos $^{(1)}$. En un porcentaje menor también se puede encontrar material genético de VEB, siendo este hallazgo más frecuente en los pacientes HIV positivos ${ }^{(5)}$. A menudo en el análisis citológico del líquido seroso predominan las células inflamatorias sin poder observarse las neoplásicas ${ }^{(14)}$, siendo en estos casos la biopsia de la membrana serosa un método complementario de extremada importancia.

En nuestro caso no pudimos realizar el diagnóstico mediante la citología del líquido pleural ni peritoneal, arribando al diagnóstico en la autopsia. Las células fueron negativas para marcadores de células B (CD79a, CD20, kappa, lambda y PAX5) y T (CD3), siendo positivas para marcadores de células plasmáticas (CD138) y 
linfocitos activados (CD30). La positividad de ADN VHH-8 se obtuvo mediante inmunohistoquímica permitiendo la confirmación diagnóstica. EI VEB fue negativo como la mayoría de casos no asociados a HIV.

Dada la escasa cantidad de casos no existe un régimen terapéutico estandarizado, aunque la terapia más utilizada suele ser etopósido, doxorrubicina, ciclofosfamida, vincristina y prednisona (EPOCH) asociado a TARV en HIV positivo ${ }^{(1)}$.

El pronóstico es malo y puede estar relacionado con la cantidad de serosas comprometidas, siendo peor a mayor cantidad de cavidades involucradas $^{(14)}$. La mediana de supervivencia global suele ser de 6 meses en pacientes HIV negativos y 40 meses para HIV positivos ${ }^{(1)}$. La causa de muerte más frecuente es debida a la progresión del derrame.

\section{CONCLUSIÓN}

El LEP es un LNH de escasa frecuencia que se caracteriza por derrame linfomatoso maligno en las cavidades serosas. Afecta preferentemente a individuos HIV positivos, pero la ausencia de infección por HIV no debe descartar la enfermedad. El compromiso extracavitario debe ser buscado aún en pacientes asintomáticos.

\section{Limitaciones de responsabilidad:}

La responsabilidad de este trabajo es exclusivamente de los autores.

\section{Conflicto de interés:}

Ninguno

\section{Fuentes de apoyo:}

La presente investigación no contó con fuentes de financiación.

\section{Originalidad:}

Este artículo es original y no ha sido enviado para su publicación a otro medio de difusión científica en forma completa ni parcialmente.

\section{Cesión de derechos:}

Los participantes de este trabajo ceden el derecho de autor a la Universidad Nacional de Córdoba para publicar en la RFCM y realizar las traducciones necesarias.

\section{Contribucion de los autores:}

Todos los autores han participado en la concepción del diseño, recolección de la información y elaboración del manuscrito, haciéndose públicamente responsables de su contenido y aprobando su versión final.

\section{BIBLIOGRAFÍA}

1. Hu Z, Pan Z, Chen W, Shi Y, Wang W, Yuan J, Wang E, Zhang $S$, Kurt $H$, Mai $B$, Zhang $X$, Liu $H$, Rios $A A$, Ma HY, Nguyen ND, Medeiros LJ, $\mathrm{Hu}$ S. Primary Effusion Lymphoma: A Clinicopathological Study of 70 Cases. Cancers (Basel). 2021 Feb 19;13(4):878. doi: 10.3390/cancers13040878.

2. Lurain K, Polizzotto MN, Aleman K, Bhutani M, Wyvill KM, Gonçalves PH, Ramaswami R, Marshall VA, Miley W, Steinberg $S M$, Little RF, Wilson W, Filie AC, Pittaluga S, Jaffe ES, Whitby D, Yarchoan R, Uldrick TS. Viral, immunologic, and clinical features of primary effusion lymphoma. Blood. 2019 Apr 18;133(16):1753-1761. doi: 10.1182/blood-2019-01-893339.

3. Dhungel BM, Montgomery ND, Painschab MS, Mulenga M, Tomoka T, Kaimila B, Zuze T, Kasonkanji E, Kampani C, Chimzimu $F$, Randall C, Krysiak $R$, Seguin R, Fedoriw $Y$, Gopal $S$. 'Discovering' primary effusion lymphoma in Malawi. AIDS. $2018 \mathrm{Sep}$ 24;32(15):2264-2266. doi: 10.1097/QAD.0000000000001933.

4. Asis O, Agusti J, Martinez Ciarpaglini C, Fernández Izquierdo A. Linfoma primitivo de cavidades extracavitario. Presentación de un caso. Revista De La Facultad De Ciencias Médicas De Córdoba. 2018; Suplemento JIC XIX:137-138.
5. Kim Y, Leventaki V, Bhaijee F, Jackson CC, Medeiros LJ, Vega $F$. Extracavitary/solid variant of primary effusion lymphoma. Ann Diagn Pathol. 2012 Dec;16(6):441-6. doi: 10.1016/j.anndiagpath.2012.03.004.

6. Saggini A, Di Prete M, Facchetti S, Rapisarda VM, Anemona L. Panniculitis-Like Presentation of Extracavitary Primary Effusion Lymphoma. Am J Dermatopathol. 2020 Jun;42(6):446-451. doi: 10.1097/DAD.0000000000001539.

7. Boulanger E, Agbalika F, Maarek O, Daniel MT, Grollet L, Molina JM, Sigaux F, Oksenhendler E. A clinical, molecular and cytogenetic study of 12 cases of human herpesvirus 8 associated primary effusion lymphoma in HIV-infected patients. Hematol J. 2001;2(3):172-9. doi: 10.1038/sj.thj.6200096.

8. Nador RG, Cesarman E, Chadburn A, Dawson DB, Ansari MQ, Sald J, Knowles DM. Primary effusion lymphoma: a distinct clinicopathologic entity associated with the Kaposi's sarcomaassociated herpes virus. Blood. 1996 Jul 15;88(2):645-56.

9. Kaplan LD. Human herpesvirus-8: Kaposi sarcoma, multicentric Castleman disease, and primary effusion lymphoma. Hematology Am Soc Hematol Educ Program. 2013;2013:103-8. doi: 10.1182/asheducation-2013.1.103.

10. Paner GP, Jensen J, Foreman KE, Reyes CV. HIV and HHV-8 negative primary effusion lymphoma in a patient with hepatitis $C$ virus-related liver cirrhosis. Leuk Lymphoma. 2003 Oct;44(10):18114. doi: 10.1080/1042819031000104015.

11. Hsieh PY, Huang SI, Li DK, Mao TL, Sheu JC, Chen CH. Primary effusion lymphoma involving both pleural and abdominal cavities in a patient with hepatitis $B$ virus-related liver cirrhosis. J Formos Med Assoc. 2007 Jun;106(6):504-8. doi: 10.1016/S09296646(09)60302-8.

12. Jones D, Ballestas ME, Kaye KM, Gulizia JM, Winters GL, Fletcher J, Scadden DT, Aster JC. Primary-effusion lymphoma and Kaposi's sarcoma in a cardiac-transplant recipient. N Engl J Med. 1998 Aug 13;339(7):444-9. doi: 10.1056/NEJM199808133390705.

13. Sanjay $P$, Philip X. Primary Effusion Lymphoma. Arch Pathol Lab Med. 2013;137(8):1152-1154. doi: 10.5858/arpa.2012-0294-RS.

14. Rossi G, Cozzi I, Della Starza I, De Novi LA, De Propris MS, Gaeta A, Petrucci L, Pulsoni A, Pulvirenti F, Ascoli V. Human herpesvirus-8-positive primary effusion lymphoma in HIV-negative patients: Single institution case series with a multidisciplinary characterization. Cancer Cytopathol. 2021 Jan;129(1):62-74. doi: 10.1002/cncy.22344.

15. Chadburn A, Hyjek E, Mathew S, Cesarman E, Said J, Knowles $D M$. KSHV-positive solid lymphomas represent an extra-cavitary variant of primary effusion lymphoma. Am J Surg Pathol. 2004 Nov;28(11):1401-16. doi: 10.1097/01.pas.0000138177.10829.5c. 